\title{
El trabajo infantil en MÉxico y SUS CAUSAS
}

\author{
Pedro Orraca*
}

Fecha de recepción: 04 de septiembre de 2013. Fecha de aceptación: 31 de enero de 2014.

\section{RESUMEN}

Este artículo estudia la importancia que tiene el orden de nacimiento de los hijos y la falta de recursos económicos en el hogar sobre los niveles de asistencia escolar y la participación en el mercado laboral de la población menor de edad en México. Con base en datos del Módulo de Trabajo Infantil, se estima una serie de modelos probit multivariados bajo distintas especificaciones con el objetivo de minimizar los problemas de endogeneidad de la muestra. El análisis indica que los hijos primogénitos tienen una menor probabilidad de asistir a la escuela y una mayor propensión a participar en el mercado laboral con respecto al resto de sus hermanos. Esta relación se acentúa al enfocarnos en las familias donde el trabajo infantil surge como respuesta a la falta de recursos económicos, lo que sugiere que el resultado es producto de la mayor capacidad de ingresos de los hijos primogénitos.

Palabras clave: México, orden de nacimiento, trabajo infantil, nińos y adolescentes, mercado laboral

Clasificación JEL: C01, I21, I31, I32, J01, J13.

\section{Child Labor and its Causes in Mexico}

\begin{abstract}
This article studies the effect of birth order and low household economic resources on school attendance and labor market participation among the population of minors in Mexico. Drawing on data from the Child Labor Center, this work estimates a series of multivariate probit models with different specifications, aiming to minimize problems related to the endogeneity of the sample. The analysis reveals that first-born children are less likely to attend school and more likely to participate in the labor market than their siblings. This relationship is stronger in families where child labor is a response to a lack of economic resources, which suggests that it is the result of the greater income-earning capacity of first-born children.
\end{abstract}

Key Words: Mexico, birth order, child labor, children and teenagers, labor market.

\footnotetext{
* Universidad de Sussex, Reino Unido. p.orraca-romano@sussex.ac.uk
} 


\section{Pedro Orraca}

\section{LE TRAVAIL D'ENFANTS AU MEXIQUE ET SES CAUSES \\ Résumé}

Cet article étudie l'impact de l'ordre de naissance des enfants et du manque de ressources économiques dans le foyer sur les niveaux d'assistance à l'école et de participation au marché du travail de la population mineure au Mexique. Sur la base de données du Module de Travail Infantile, on estime une série de modèles probit multivariés selon distinctes spécifications dans le but de minimiser les problèmes d'endogénéité de l'échantillon. L'analyse indique que la probabilité que l'enfant aîné aille à l'école est inférieure et la propension à ce qu'il participe au marché du travail supérieure par rapport à ses frères et sœurs. Cette relation s'accentue quand on se centre sur les familles où le travail des enfants surgit comme réponse au manque de ressources économiques, ce qui suggère que le résultat est produit de la majeure capacité de revenus des aînés.

Mots clés : Mexique, ordre de naissance, travail des enfants, enfants et adolescents, marché du travail.

\section{O TRABALHO INFANTIL NO MÉXICO E SUAS CAUSAS}

\section{Resumo}

Neste artigo se estuda a importância que tem a ordem de nascimentos dos filos e a falta de recursos económicos no lar sobre os níveis de assistência escolar e a participação no mercado laboral da população menor de idade no México. Com base em dados do Módulo de Trabalho Infantil, se estima uma série de modelos probit multivariados sob distintas especificaçóes com o objetivo de minimizar os problemas de endogeneidade da amostra. A análise indica que os filhos primogênitos têm uma menor probabilidade de assistir à escola e uma maior propensão a participar do mercado laboral quando comparados com seus irmãos. Esta relação é mais forte ao estudar as famílias onde o trabalho infantil surge cmo resposta à falta de recursos econômicos, o que sugere que o resultado é produto da maior capacidade de gerar renda dos filhos primogênitos.

Palavras-chave: México, ordem de nascimento, trabalho infantil, crianças e adolescentes, mercado laboral.

\section{墨西哥童工及其根源}

摘要

本文研究了墨西哥低龄人口中的出生顺序与低收入家庭经济资源对入学和 劳动力市场参与的影响。该项研究利用儿童劳动力中心数据，根据不同的 情景, 建立了多元概率比回归模型, 目标是最小化样本的内生性问题。研 究显示, 第一个出生的孩子与其兄弟姐妹相比, 入学的可能性较低, 而参 与劳动力市场的可能性较高。这种关系在经济资源缺乏的家庭更加明显, 这表明第一个出生的孩子有着更大的创收能力, 使其成为童工的可能性较 大。

关键词：墨西哥出生顺序童工儿童和青年劳动力市场 


\section{INTRODUCCIÓN}

El problema del trabajo infantil es un fenómeno en escalas mundial y nacional. La Organización Internacional del Trabajo (оІт) estima que en 2008, más de 306 millones de individuos entre 5 y 17 años de edad participaron en el mercado laboral (oit, 2010: 7). Sólo en México, se calcula que en 2011 el número total de niños ocupados ascendió a 3.0 millones (INEGI, 2012).

Este suceso es un problema porque suele impedir que los niños y adolescentes alcancen un desarrollo pleno, al limitar su acumulación de capital humano y reducir su nivel de ingresos a lo largo del ciclo de vida laboral. En el caso de México, esto adquiere mayor importancia debido a que la educación se caracteriza por tener altos rendimientos privados, lo que deja en una amplia desventaja a la población con bajos niveles educativos. Asimismo, es probable que estos efectos se extiendan a generaciones futuras debido al bajo grado de movilidad social que prevalece en el país. En el nivel agregado, el trabajo infantil suele ser visto como indeseable al disminuir el acervo de capital humano en el mediano y largo plazos, lo que limita los niveles de crecimiento económico futuros.

A diferencia de lo que normalmente se piensa, el trabajo infantil no sólo es producto del egoísmo de los padres, sino que surge a partir de la estrategia del hogar para sobrevivir y obtener una mayor cantidad de bienes. El trabajo de los niños y adolescentes es un síntoma de otros problemas en el país, como lo son la presencia de sistemas educativos deficientes, actos de discriminación dentro de las familias y la falta de oportunidades para ciertos sectores de la población. Esto se manifiesta en el hecho de que en las economías desarrolladas raramente se presentan casos de trabajo infantil (Basu y Van, 1998).

El presente artículo tiene como objetivo estudiar el efecto del orden de nacimiento de los hijos y la falta de recursos económicos en el hogar sobre la asignación de tiempo destinado a la asistencia escolar, el trabajo remunerado y el no remunerado entre la población menor de edad en México. El país representa un caso interesante porque se caracteriza por tener un nivel de ingreso medio-alto, un elevado grado de desigualdad, niveles significativos de pobreza y trabajo infantil, y distintos programas sociales de alto alcance cuyos objetivos directos e indirectos son promover el desarrollo humano de la población.

Aquí se amplía la escasa literatura que analiza el efecto del orden de nacimiento de los hijos sobre los niveles de asistencia escolar y la participación en el mercado laboral para el caso de México. A diferencia de gran parte de las investigaciones pasadas, ésta no se limitará a las personas de 12 años de edad en adelante, sino que se enfocará en la población de entre 6 y 16 años. Adicionalmente, se distingue entre las actividades de trabajo remuneradas y 
no remuneradas, mientras que el análisis econométrico toma en cuenta las interrelaciones de estas decisiones laborales con la asistencia escolar. Finalmente, se proporciona evidencia actualizada sobre el panorama del trabajo infantil en escala nacional.

Entre los resultados se destaca la presencia de diferencias importantes en la asignación de tiempo de las actividades de los hijos de acuerdo a su orden de nacimiento. Se observa que los hijos de mayor edad tienen una probabilidad menor de asistir a la escuela y son más propensos a incursionar en el mercado laboral comparados con el resto de sus hermanos. Por otro lado, los hijos de menor edad tienen una inclinación mayor a asistir a la escuela, y en el caso de las mujeres, una propensión menor a incurrir en actividades de trabajo remunerado. Las relaciones encontradas se acentúan al enfocarnos en los hogares donde los hijos laboran debido a la falta de recursos. Estos hallazgos contradicen lo que normalmente se observa en países desarrollados, donde el orden de nacimiento tiende a favorecer a los hijos de mayor edad. En México, los efectos del orden de nacimiento sobre las asignaciones de tiempo entre actividades escolares y laborales tienden a favorecer a los hijos de menor edad.

\section{EL TRABAJO INFANTIL EN MÉXICO}

La Constitución política de los Estados Unidos Mexicanos estipula en su artículo 123 que está prohibida la utilización del trabajo de los menores de 14 años (CPeum, artículo 123, apartado A, fracción III). Por su parte, la Ley Federal del Trabajo establece en sus artículos 22 y 23 que no está permitido que trabajen los menores de 14 ańos y los mayores de esta edad y menores de 16 años que no hayan terminado su educación obligatoria, salvo casos excepcionales. Sin embargo, a pesar de que hay un marco legal para ahuyentar el trabajo infantil, los niveles de participación económica de esta cohorte son significativos. De acuerdo a datos del Módulo de Trabajo Infantil (MTI) (INEGI, 2012), 10.5\% de la población de entre 5 y 17 ańos de edad trabajaba en 2011. De éstos, $70.9 \%$ se encuentra dentro de la edad legal para laborar, lo que implica que más de 882000 individuos, entre los 5 y 13 años, participan en el mercado de trabajo.

Asimismo, a partir de estas cifras se observa un alto grado de heterogeneidad según la entidad federativa de origen, el género, el tipo de trabajo desempeñado y el tamaño de la localidad de residencia. Esto se puede ver en el hecho de que mientras en Chihuahua 4.2\% de la población menor de edad trabaja, en Guerrero $26.5 \%$ de los niños y adolescentes participan en 
el mercado laboral. En escala nacional, $67.9 \%$ de los trabajadores infantiles son varones, mientras que $48.3 \%$ de los menores que trabajan reportan que no reciben ninguna remuneración por sus tareas. Adicionalmente, el trabajo infantil tiende a ser más común en las zonas rurales que en las urbanas, donde $37.5 \%$ del total de los trabajadores infantiles reside en localidades con menos de 2500 habitantes.

La participación en el mercado laboral implica una asignación de tiempo al trabajo en perjuicio de las actividades escolares. Si bien la tasa de asistencia escolar entre la población de 5 a 17 años es de $91.1 \%$, ésta se reduce considerablemente al enfocarse en los menores que trabajan, donde sólo $60.9 \%$ de la población que labora también estudia. Finalmente, $4.8 \%$ del total de los menores de edad no estudian ni trabajan.

\section{TEORÍA Y EVIDENCIA EMPÍRICA}

\section{Teoría}

La oferta laboral de los niños y adolescentes se determina en función de las características económicas y demográficas del hogar. Entre éstas se incluyen la edad, el género y el nivel educativo de sus miembros, el número de hermanos que habita en la vivienda, y los costos y rendimientos de los distintos usos de tiempo (Bando, López-Calva et al., 2005: 4).

Estudios que analizan el trabajo infantil y la asistencia escolar tienden a sustentarse en modelos neoclásicos de decisiones del hogar o suelen ligar dichos fenómenos con las decisiones de fertilidad de la madre (Bando, LópezCalva et al., 2005; Dammert, 2010). En dichos modelos, padres altruistas determinan la asignación de tiempo de sus hijos, donde las familias se esfuerzan por lograr un consumo mínimo de subsistencia antes de obtener otros bienes o realizar algún tipo de inversión (e.g. Basu y Van, 1998; Baland y Robinson, 2000; Cigno y Rosati, 2005). Al ser percibidos como activos, las inversiones en los hijos se realizan hasta el punto en donde el costo marginal de la educación es igual al beneficio marginal, tomando en cuenta el costo de oportunidad de no trabajar (Dammert, 2010: 200). Debido a la presencia de costos marginales crecientes ligados a las inversiones en capital humano, hay una relación inversa entre la demanda por la calidad y la cantidad de hijos en el hogar (Becker, 1991). ${ }^{1}$

Por una parte, se puede pensar que en los hogares con bajos ingresos los hijos primogénitos tienen una mayor inclinación a trabajar, dado que su expe- 
riencia y acervo de capital humano, respecto al resto de sus hermanos, les permite realizar actividades más complejas, con lo que alcanzan elevados niveles de productividad, obteniendo así mayores sueldos y más recursos para el hogar. Este ingreso adicional puede relajar las restricciones presupuestarias del hogar, haciendo posible que el resto de los hijos asistan a la escuela (Emerson y Souza, 2008: 1649). Por ello, si el trabajo infantil surge por razones de subsistencia, se esperaría una mayor participación entre los hijos que nacieron primero.

Por otro lado, Dammert (2010: 200) argumenta que los factores biológicos también desempeñan un papel importante en las asignaciones de tiempo dentro del hogar. Específicamente, se arguye que al momento que la madre tiene sus últimos hijos, debido a su edad más avanzada, los niños al nacer en promedio pesan menos, lo que está correlacionado con menores niveles de habilidad en el mediano y largo plazos.

Con base en lo anterior, se puede pensar que si el trabajo y la asistencia escolar no responden a diferencias en los niveles de experiencia, sino a discrepancias en la escala de habilidades entre los hijos, entonces el efecto del orden de nacimiento no es del todo claro. Si bien un mayor nivel de habilidad se refleja en el corto plazo un mayor costo de oportunidad de estudiar, también es cierto que esto se puede reflejar en mayores retornos a la educación en el largo plazo y, por lo tanto, en caso de que se trabaje, una mayor pérdida económica respecto al resto de los hermanos (Emerson y Souza, 2008: 1648).

Por ello, el hecho de que el niño o adolescente asista a la escuela o participe en el mercado laboral depende de las diferencias salariales entre los distintos hijos, y de la valoración que el hogar le asigne al consumo familiar presente, en escala de bienestar a mediano y largo plazos del hijo y a la acumulación de capital humano, entre otros (Emerson y Souza, 2008: 1649). Esto hace que la relación entre el orden de nacimiento y la asignación de tiempo entre actividades escolares y laborales sea ante todo una cuestión empírica.

\section{Evidencia empírica}

Diversos estudios se han enfocado en las decisiones laborales y educativas de la población menor de edad en México. Entre éstos, K. Córdova (2009) analiza el efecto del orden de nacimiento de los hijos sobre los años de escolaridad y la asignación de tiempo destinado a distintas actividades como la recreación, los quehaceres del hogar y el cuidado de otros familiares. Con base en datos de la Encuesta Nacional sobre Niveles de Vida de los Hogares (ennviH), la investigadora emplea el método en dos etapas planteado por Heckman (1979) 
para estimar el efecto del orden de nacimiento sobre los años de educación, mientras que para conocer su efecto sobre las asignaciones de tiempo estima un modelo mediante el método de mínimos cuadrados ordinarios (MCO). Entre los resultados no se encuentra evidencia de que el orden de nacimiento esté relacionado con el número de años de escolaridad; sin embargo, sí se observa que el orden de nacimiento y el género tienen un efecto significativo sobre el tiempo destinado a las actividades del hogar y al cuidado de sus miembros. Por otra parte, A. López (2005) analiza de manera conjunta los determinantes de la participación escolar y laboral de la población de 6 a 17 años de edad. Con base en la Encuesta Nacional de Ingresos y Gastos de los Hogares (ENIGH), A. López estima un modelo probit ordinal, donde los individuos eligen entre asistir a la escuela, trabajar medio tiempo, o trabajar tiempo completo. Entre los resultados se encuentra que el nivel de ingreso y los activos del hogar incrementan la probabilidad de que los menores asistan a la escuela, mientras que las restricciones crediticias que enfrenta el hogar la reducen.

Adicionalmente, Levison; Moe et al. (2001) estudian los determinantes de la asistencia escolar y la participación laboral para la población de entre 12 y 17 años. Para ello, los autores emplean información proveniente de la Encuesta Nacional de Empleo Urbano (ENEU), mientras que para el ejercicio empírico estiman un modelo logit multinomial. Entre los resultados se observa que las mujeres tienen una mayor probabilidad de asistir a la escuela; sin embargo, si en la definición de trabajo se incluyen las actividades del hogar, las mujeres muestran una propensión menor a especializarse en los estudios respecto a los hombres. Por otro lado, López-Calva y Freije (2001) analizan la relación entre el trabajo infantil y una serie de variables en las cuales se incluyen: la situación de pobreza del hogar, la tasa de desempleo de los padres, el salario y la aceptación social del trabajo, entre otras. Por medio de información longitudinal de la ENEu para México y de la Encuesta de Hogares por Muestreo (ЕHм) para Venezuela, los autores emplean una serie de modelos y concluyen que la situación de pobreza y el nivel de incidencia son determinantes robustos del trabajo infantil, mientras que el salario y el desempleo no lo son.

Por otra parte, diversos estudios se han enfocado en analizar el efecto del programa Progresa, posteriormente renombrado Oportunidades, sobre la asistencia escolar y el trabajo infantil. Dentro de estos, destaca la investigación realizada por Skoufias y Parker (2001), donde con base en distintas encuestas levantadas, con el propósito de evaluar el efecto del programa, encuentran que éste repercutió considerablemente sobre las dos variables, al incrementar, de manera significativa, la asistencia escolar y reducir el trabajo infantil. En un estudio similar, pero enfocándose primordialmente en la población indígena, 
Bando; López-Calva et al. (2005) argumentan que el programa tuvo un efecto positivo sobre el trabajo infantil, al disminuir su incidencia en $8 \%$ entre los años 1997 y 2000 . Asimismo, se observa que el trabajo infantil está ligado a las decisiones de fertilidad de la madre y al mercado laboral de la población adulta, mientras que la educación del padre está positivamente relacionada con la probabilidad de que los nińos asistan a la escuela y negativamente relacionada con la propensión a trabajar de la población menor de edad.

Respecto a la literatura internacional, Dammert (2010) analiza cómo repercute de la diferencia de género entre hermanos y el orden de nacimiento de los hijos sobre los niveles de trabajo, las labores domésticas y la asistencia escolar para el caso de Guatemala y Nicaragua. El estudio encuentra que los varones que nacen primero destinan una mayor cantidad de tiempo al trabajo y a las actividades del hogar, mientras que las hijas primogénitas tienen una mayor inclinación hacia las labores domésticas comparado con el resto de sus hermanos. En una investigación similar para el caso de Ecuador, De Haan; Plug et al. (2012) observan que el nivel de escolaridad aumenta junto con el orden de nacimiento, mientras que los niveles de trabajo infantil decrecen con éste. Por último, Emerson y Souza (2008) se concentran en el caso de Brasil, donde encuentran que los hijos más chicos tienen una menor probabilidad de trabajar, mientras que los primogénitos tienen una mayor propensión a participar en el mercado laboral y una menor probabilidad de asistir a la escuela.

\section{Datos}

El estudio se sustenta en datos del MTI, recabados durante el cuarto trimestre de 2011 como anexo a la Encuesta Nacional de Ocupación y Empleo (ENOE). El MTI complementa la información normalmente recolectada para la población de 12 a 17 años en la ENOE y extiende el análisis al captar las características referentes a las actividades escolares y laborales que realizan los individuos entre las edades de 5 y 11 años. Esto permite identificar los determinantes de la participación en el mercado laboral y la asistencia escolar de la población mexicana menor de edad.

De acuerdo con el MTI, se define como trabajador infantil a todo aquel individuo entre los 5 y 17 años que durante la semana anterior a la entrevista realizó alguna actividad económica o estaba por incorporarse a una de manera inminente. Por actividad económica se entiende la producción para el consumo particular o toda aquella acción realizada con la intención de producir o proporcionar bienes y servicios para el mercado. ${ }^{2} \mathrm{El}$ cuestionario fue aplicado 
a más de 96000 individuos en las 32 entidades federativas del país. El módulo incluye información, tanto de áreas urbanas como rurales y es representativo en escala nacional.

El análisis se restringe a la población de entre 6 y 16 años de edad, lo cual permite enfocarse en los menores que se encuentran entre las edades de estudio obligatorio. Sólo se incluyen viviendas donde habitan tanto la madre como el padre, con el propósito de minimizar los efectos generados por diferencias estructurales en las características del hogar. El estudio se limita a los hijos nacidos en México y de padres mexicanos, con el fin de suprimir la presencia de discrepancias culturales entre la población nativa y extranjera que reside en el país.

Por último, se clasifica como trabajadores infantiles a los menores que reportan un número positivo de horas laboradas, y se separa a los individuos de acuerdo a su género y estatus como trabajadores remunerados o no. La muestra final está compuesta por 46613 individuos, de los cuales 23765 son hombres y 22848 son mujeres.

\section{Modelo}

Si bien los niños y adolescentes distribuyen su tiempo entre numerosas actividades, nos enfocamos de manera exclusiva en la asignación de tiempo destinado a la asistencia escolar, actividades de trabajo remuneradas y labores no remuneradas. Para ello, se estima un modelo probit multivariado donde se asume que la decisiones de asignación de tiempo, entre estas tres tareas, están interrelacionadas, aun cuando individualmente se tomen de manera separada.

La estructura del modelo probit multivariado es similar a la de un sistema de ecuaciones aparentemente incorrelacionadas, con la diferencia notoria de que en el presente análisis la variable dependiente es dicotómica. Considere un modelo probit multivariado de tres ecuaciones:

$$
\begin{aligned}
& y_{i, m}^{*}=\beta_{m}^{\prime} x_{i, m}+\varepsilon_{i, m}, m=1,2,3 \\
& y_{i, m}=1, \text { si } y_{i, 1}^{*}>0 \text { y } 0 \text { en otro caso }
\end{aligned}
$$

donde $y_{i, m}^{*}$ representa una variable latente subyacente vinculada a $y_{i, m}$, la cual constituye una variable dicotómica que toma el valor de uno si el evento ocurre y cero en otro caso. ${ }^{3}$ Conjuntamente, se asume que los errores $\varepsilon_{i, m}$ siguen una distribución normal multivariada, con media cero y una matriz 


\section{Pedro Orraca}

de varianza-covarianza $V$ que contiene valores de uno en la diagonal y correlaciones $\rho_{j, k}=\rho_{k, j}$ fuera de ella. $X_{i, m}$ denota una matriz de variables exógenas que contiene la edad de los niños, de los padres, y el nivel de estudios de los segundos, entre otras. Dentro de $X_{i, m}$ se incluyen los regresores centrales a nuestro estudio que son una variable dicotómica que toma el valor de uno si el individuo es el hijo de mayor edad entre los habitantes del hogar y cero en otro caso, y una variable dicotómica que toma el valor de uno si el niño o adolescente es el hijo de menor edad entre los distintos residentes del hogar y cero en otro caso. En ambas ocasiones, el grupo de referencia está conformado por el resto de los hermanos que habitan en el mismo hogar.

La estructura normal multivariada del término de error lleva a que incluso después de controlar, por una serie de variables explicativas, las decisiones de interés estén interrelacionadas. Sin embargo, un punto débil del modelo es que asume la misma estructura de correlación entre cualquier par de decisiones para todos los individuos, lo cual representa un supuesto altamente restrictivo. Por otro lado, el modelo tiene la ventaja de que no requiere incluir el mismo conjunto de regresores en cada ecuación, aunque dada la naturaleza del análisis en este caso optamos por hacerlo. Finalmente, el modelo es estimado mediante el método de máxima verosimilitud simulada, mediante el simulador Geweke-Hajivassiliou-Keane (e.g. Börsch-Supan y Hajivassiliou, 1993; Keane, 1994). ${ }^{4}$

\section{RESULTADOS}

\section{Estadística descriptiva}

El cuadro 1 presenta la media y la desviación estándar de las principales variables empleadas en el estudio. Se observa que 94.4 de los niños y $95.4 \%$ de las niñas asisten a la escuela. Adicionalmente, hay diferencias importantes en la proporción de trabajadores de acuerdo al género, ya que mientras $9.9 \%$ de los varones incurren en actividades laborales, sólo 4.6\% de las mujeres trabajan. Esta relación se mantiene al dividir la muestra entre individuos que incurren en trabajo remunerado o no, o entre aquellos que laboran más de 15 horas o quienes no lo hacen. Se observa que tanto los padres como las madres de los menores son gente joven, con un promedio de edad de 40.2 y 37.0 años, respectivamente. Por otra parte, $28.9 \%$ de los hogares analizados pertenecen a comunidades rurales. 
Cuadro 1. Estadística descriptiva

\begin{tabular}{|c|c|c|c|c|}
\hline & \multicolumn{2}{|c|}{ Hombres } & \multicolumn{2}{|c|}{ Mujeres } \\
\hline & Media & $\begin{array}{l}\text { Desviación } \\
\text { estándar }\end{array}$ & Media & $\begin{array}{l}\text { Desviación } \\
\text { estándar }\end{array}$ \\
\hline Edad & 10.8900 & 3.1220 & 10.8800 & 3.1050 \\
\hline Asiste a la escuela & 0.9444 & 0.2290 & 0.9542 & 0.2089 \\
\hline Trabaja & 0.0989 & 0.2986 & 0.0455 & 0.2087 \\
\hline \multicolumn{5}{|l|}{ Trabajo remunerado } \\
\hline$>0$ horas & 0.0446 & 0.2064 & 0.0205 & 0.1419 \\
\hline$>15$ horas & 0.0300 & 0.1706 & 0.0120 & 0.1093 \\
\hline \multicolumn{5}{|l|}{ Trabajo no remunerado } \\
\hline$>0$ horas & 0.0543 & 0.2267 & 0.0250 & 0.1563 \\
\hline$>15$ horas & 0.0265 & 0.1608 & 0.0116 & 0.1073 \\
\hline Rural & 0.2844 & 0.4511 & 0.2943 & 0.4557 \\
\hline Número de hermanos & 2.0120 & 1.3210 & 2.0210 & 1.3260 \\
\hline Hijo mayor & 0.3955 & 0.4889 & 0.3969 & 0.4892 \\
\hline Hijo menor & 0.3731 & 0.4836 & 0.3706 & 0.4829 \\
\hline Diferencia de edad respecto al hijo mayor & 3.4130 & 3.9770 & 3.4970 & 4.1230 \\
\hline Diferencia de edad respecto al hijo menor & 3.6960 & 3.8380 & 3.6990 & 3.8220 \\
\hline Diferencia de edad entre mayor y menor & 2.6780 & 1.5370 & 2.7100 & 1.5630 \\
\hline Edad del padre & 40.1600 & 7.8820 & 40.2200 & 8.0220 \\
\hline Edad de la madre & 36.9400 & 6.7930 & 37.1100 & 6.9500 \\
\hline Años de educación del padre & 8.4490 & 4.5980 & 8.3890 & 4.6750 \\
\hline Años de educación de la madre & 7.9820 & 5.4020 & 7.9540 & 5.3630 \\
\hline Observaciones & \multicolumn{2}{|c|}{23765} & \multicolumn{2}{|c|}{22848} \\
\hline
\end{tabular}

Fuente: Elaboración propia con base en datos del MTI-ENOE, 2011.

Para ver si hay diferencias considerables en la población estudiada, se analizó la asistencia escolar y la actividad laboral por rangos de edad. El cuadro 2 muestra que a medida que los individuos crecen, la proporción que se en- 


\section{Pedro Orraca}

cuentra estudiando se reduce, donde su máximo radica entre las edades de 6 y 10 , para posteriormente disminuir hasta los 16 años. Esto corresponde a la atrición natural que ocurre al incrementar el grado escolar. Respecto al trabajo remunerado y el no pagado se presenta la situación inversa, donde aquéllos con mayores probabilidades de incurrir en actividades laborales se concentran entre las edades de 15 y 16 ańos. Lo anterior denota la presencia de una relación positiva entre la edad de los menores y su propensión a trabajar.

Cuadro 2. Trabajo infantil y asistencia escolar por rango de edad

Hijo mayor

\begin{tabular}{|c|c|c|c|c|c|c|}
\hline \multirow[b]{2}{*}{ Edad } & \multicolumn{3}{|c|}{ Hombres } & \multicolumn{3}{|c|}{ Mujeres } \\
\hline & $\begin{array}{c}\text { Asiste a la } \\
\text { escuela }\end{array}$ & $\begin{array}{c}\text { Trabajo } \\
\text { remunerado }\end{array}$ & $\begin{array}{l}\text { Trabajo no } \\
\text { remunerado }\end{array}$ & $\begin{array}{c}\text { Asiste a la } \\
\text { escuela }\end{array}$ & $\begin{array}{c}\text { Trabajo } \\
\text { remunerado }\end{array}$ & $\begin{array}{c}\text { Trabajo no } \\
\text { remunerado }\end{array}$ \\
\hline $6-8$ & 0.9960 & 0.0154 & 0.0127 & 0.9933 & 0.0033 & 0.0045 \\
\hline $9-10$ & 0.9910 & 0.0158 & 0.0287 & 0.9962 & 0.0119 & 0.0272 \\
\hline $11-12$ & 0.9881 & 0.0270 & 0.0625 & 0.9816 & 0.0107 & 0.0235 \\
\hline $13-14$ & 0.9292 & 0.0574 & 0.0764 & 0.9372 & 0.0241 & 0.0417 \\
\hline $15-16$ & 0.8109 & 0.1474 & 0.1033 & 0.8272 & 0.0659 & 0.0503 \\
\hline
\end{tabular}

Hijo menor

\begin{tabular}{|c|c|c|c|c|c|c|}
\hline \multirow[b]{2}{*}{ Edad } & \multicolumn{3}{|c|}{ Hombres } & \multicolumn{3}{|c|}{ Mujeres } \\
\hline & $\begin{array}{c}\text { Asiste a la } \\
\text { escuela }\end{array}$ & $\begin{array}{c}\text { Trabajo } \\
\text { remunerado }\end{array}$ & $\begin{array}{l}\text { Trabajo no } \\
\text { remunerado }\end{array}$ & $\begin{array}{c}\text { Asiste a la } \\
\text { escuela }\end{array}$ & $\begin{array}{c}\text { Trabajo } \\
\text { remunerado }\end{array}$ & $\begin{array}{l}\text { Trabajo no } \\
\text { remunerado }\end{array}$ \\
\hline $6-8$ & 0.9877 & 0.0061 & 0.0105 & 0.9944 & 0.0040 & 0.0056 \\
\hline $9-10$ & 0.9896 & 0.0073 & 0.0307 & 0.9962 & 0.0158 & 0.0155 \\
\hline $11-12$ & 0.9858 & 0.0204 & 0.0447 & 0.9883 & 0.0093 & 0.0269 \\
\hline $13-14$ & 0.9452 & 0.0427 & 0.0748 & 0.9375 & 0.0135 & 0.0231 \\
\hline $15-16$ & 0.8303 & 0.1338 & 0.0710 & 0.8814 & 0.0503 & 0.0359 \\
\hline
\end{tabular}

Fuente: Elaboración propia con base en datos del MTI-ENOE, 2011. 
El cuadro 3 describe los motivos por los cuales los niños y adolescentes participan en el mercado laboral. Mientras que $18.4 \%$ de los varones primogénitos trabajan debido a la falta de recursos, sólo $10.4 \%$ de los hijos más jóvenes laboran por las mismas razones. Esta situación se mantiene para ambos géneros así como para el trabajo remunerado y el no pagado y ofrece evidencia tangencial de que respecto a los otros hijos, la decisión de trabajar del hijo primogénito responde, en mayor medida, a la falta de recursos en el hogar.

Cuadro 3. Motivo por el cual se trabaja

\begin{tabular}{|c|c|c|c|c|}
\hline \multirow[t]{3}{*}{ Hijo } & \multicolumn{4}{|c|}{ Trabajo remunerado } \\
\hline & \multicolumn{2}{|c|}{ Hombres } & \multicolumn{2}{|c|}{ Mujeres } \\
\hline & Falta de recursos & Otro motivo & Falta de recursos & Otro motivo \\
\hline Mayor & 18.37 & 81.63 & 13.74 & 86.26 \\
\hline Menor & 10.41 & 89.59 & 13.12 & 86.88 \\
\hline \multirow[t]{3}{*}{ Hijo } & \multicolumn{4}{|c|}{ Trabajo no remunerado } \\
\hline & \multicolumn{2}{|c|}{ Hombres } & \multicolumn{2}{|c|}{ Mujeres } \\
\hline & Falta de recursos & Otro motivo & Falta de recursos & Otro motivo \\
\hline Mayor & 53.79 & 46.21 & 62.57 & 37.43 \\
\hline Menor & 51.98 & 48.02 & 47.29 & 52.71 \\
\hline
\end{tabular}

Fuente: Elaboración propia con base en datos del MTI-ENOE, 2011. Personas que se catalogan que trabajan por falta de recursos incluye a los menores de edad que respondieron que laboran ya sea porque el hogar necesita de su aportación económica o porque el hogar necesita de su trabajo.

\section{Resultados econométricos}

Para analizar el efecto del orden de nacimiento sobre la asistencia escolar y la participación laboral se estima un modelo probit multivariado de tres ecuaciones. El modelo incluye las variables dicotómicas de interés que indican si el individuo es el hijo de menor o mayor edad, así como una serie de controles exógenos que influyen sobre las variables dependientes. Entre éstas se incluye la edad, el grado escolar y la situación laboral de los padres, el número de hijos en el hogar, la diferencia de edad promedio entre los hermanos, y una variable dicotómica que denota si el hogar se ubica en una localidad urbana o rural. ${ }^{5}$ 
El cuadro 4 presenta los resultados para la muestra completa. La edad del niño tiene un efecto negativo sobre la asistencia escolar y una influencia positiva sobre la participación laboral. El efecto se presenta para ambos géneros, y es mayor sobre el trabajo remunerado que el no pagado. Esto refleja la relación positiva que hay entre la productividad de los menores y su edad, mientras que su inclusión permite estimar los efectos netos del orden de nacimiento una vez que se haya controlado a causa de la edad.

Respecto a las variables centrales del análisis, los varones primogénitos tienen una probabilidad menor de asistir a la escuela comparado al resto de sus hermanos, aunque la discrepancia con respecto a los hijos más chicos es mínima. Sin embargo, esta diferencia es considerable para el caso de las mujeres, donde las hijas más jóvenes son más propensas a asistir a la escuela, mientras que las primogénitas presentan una probabilidad sustancialmente menor de asistir. Adicionalmente, los varones más pequeños son menos proclives a tener un trabajo remunerado y tienen una probabilidad mayor de tener uno no pagado. En el caso de las niñas se presenta la situación inversa, donde el ser la última en nacer las lleva a que sean más propensas a tener un trabajo remunerado, lo que a su vez reduce la inclinación a tener uno no remunerado. Lo anterior sugiere que entre las mujeres ocurre un cierto grado de sustitución entre el trabajo remunerado y el no pagado, en los cuales se especializan las hijas más pequeñas y las primogénitas, respectivamente.

Por otro lado, se puede observar que a mayor nivel de escolaridad de los padres, más grande es la probabilidad de que los hombres y las mujeres asistan a la escuela. Adicionalmente, mientras los niveles educativos del padre tienen un efecto negativo sobre la propensión a trabajar, los efectos del grado de escolaridad de la madre son ambiguos. Estos resultados son esperados debido a que la variable de educación suele ser un buen indicador del nivel de ingresos del hogar, lo que tiende a reducir la participación laboral de los menores. El hecho de que el padre o la madre trabajen reduce la probabilidad de que el hijo asista a la escuela y aumenta la perspectiva de que incurran en algún tipo de trabajo remunerado. Si bien estos efectos son pequeños, no muestran que haya una relación directa entre el desempleo de los padres y el trabajo infantil, lo que sugiere es que la incursión de los niños y adolescentes en el mercado laboral no surge como respuesta a la falta de empleo de los padres. Sin embargo, no se descarta que el trabajo infantil sea producto del bajo ingreso de los padres, situación que puede orillar a los hogares a la pobreza.

En cambio, se puede observar que el tener un número mayor de hijos está asociado con una menor probabilidad de asistir a la escuela y una mayor inclinación a participar en el mercado laboral. Este resultado refleja el hecho de que los hogares más grandes tienden a ser más pobres y enfrentan mayores 
Cuadro 4. Resultados. Modelo probit multivariado. Muestra completa

\begin{tabular}{|c|c|c|c|c|c|c|}
\hline \multirow{2}{*}{$\begin{array}{l}\text { Edad } \\
\text { del niño }\end{array}$} & \multicolumn{3}{|c|}{ Hombres } & \multicolumn{3}{|c|}{ Mujeres } \\
\hline & $\begin{array}{c}\text { Asiste a la } \\
\text { escuela }\end{array}$ & $\begin{array}{c}\text { Trabajo } \\
\text { remunerado }\end{array}$ & $\begin{array}{l}\text { Trabajo no } \\
\text { remunerado }\end{array}$ & $\begin{array}{c}\text { Asiste a la } \\
\text { escuela }\end{array}$ & $\begin{array}{c}\text { Trabajo } \\
\text { remunerado }\end{array}$ & $\begin{array}{l}\text { Trabajo no } \\
\text { remunerado }\end{array}$ \\
\hline \multirow[t]{2}{*}{ Edad del niño } & $-0.2183^{\star \star \star}$ & $0.1781^{\star \star \star}$ & $0.1156^{\star \star \star}$ & $-0.2326^{\star \star \star}$ & $0.1533^{\star \star \star}$ & $0.0869 * \star \star *$ \\
\hline & $(0.0004)$ & $(0.0003)$ & $(0.0003)$ & $(0.0004)$ & $(0.0005)$ & $(0.0004)$ \\
\hline \multirow{2}{*}{$\begin{array}{l}\text { Niño más } \\
\text { chico }\end{array}$} & $-0.0585^{\star \star \star}$ & $-0.0700^{\star \star \star}$ & $0.0159^{\star \star \star *}$ & $0.0081^{\star \star \star}$ & $0.0756^{\star \star \star}$ & $-0.0754^{\star \star \star}$ \\
\hline & $(0.0026)$ & $(0.0027)$ & $(0.0026)$ & $(0.0030)$ & $(0.0036)$ & $(0.0035)$ \\
\hline \multirow{2}{*}{$\begin{array}{l}\text { Niño más } \\
\text { grande }\end{array}$} & $-0.0592^{\star \star \star}$ & $0.0305^{\star \star \star}$ & $0.1547^{\star \star \star}$ & $-0.1646^{\star \star \star}$ & $0.0719^{\star * \star}$ & $0.1291^{\star \star \star}$ \\
\hline & $(0.0022)$ & (0.0023) & $(0.0023)$ & $(0.0024)$ & $(0.0030)$ & $(0.0031)$ \\
\hline \multirow{2}{*}{$\begin{array}{l}\text { Escolaridad } \\
\text { padre }\end{array}$} & $0.0832^{\star \star \star}$ & $-0.0395^{\star \star \star}$ & $-0.0416^{\star \star \star}$ & $0.0873^{\star * *}$ & $-0.0401^{\star \star *}$ & $-0.0385^{\star \star \star}$ \\
\hline & $(0.0002)$ & $(0.0002)$ & $(0.0002)$ & $(0.0002)$ & $(0.0003)$ & $(0.0003)$ \\
\hline \multirow{2}{*}{$\begin{array}{l}\text { Escolaridad } \\
\text { madre }\end{array}$} & $0.0306^{\text {*** }}$ & $-0.0012^{\star \star \star \star}$ & $0.0167^{\star \star \star \star}$ & $0.0223^{\star \star \star}$ & $0.0148^{\star \star \star}$ & $0.0247^{\star \star \star}$ \\
\hline & $(0.0003)$ & $(0.0002)$ & $(0.0002)$ & $(0.0003)$ & $(0.0003)$ & $(0.0003)$ \\
\hline \multirow[t]{2}{*}{ Edad padre } & $0.0040^{\star \star \star *}$ & $-0.0101^{\star \star \star}$ & $0.0061^{\star \star \star *}$ & $0.0030^{\star * \star}$ & $-0.0021^{\star \star \star}$ & $0.0120^{\star \star \star}$ \\
\hline & $(0.0001)$ & $(0.0001)$ & $(0.0001)$ & $(0.0001)$ & $(0.0002)$ & $(0.0002)$ \\
\hline \multirow[t]{2}{*}{ Edad madre } & $-0.0003^{*}$ & $-0.0022^{\star \star \star}$ & $0.0016^{\star \star \star}$ & $-0.0043^{\star \star \star}$ & $-0.0073^{\star \star \star}$ & 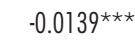 \\
\hline & $(0.0002)$ & $(0.0002)$ & $(0.0002)$ & $(0.0002)$ & $(0.0002)$ & $(0.0002)$ \\
\hline \multirow[t]{2}{*}{ Trabaja padre } & $-0.0022^{\star \star \star}$ & $0.0008^{\star \star \star}$ & $0.0074^{\star \star \star}$ & $-0.0014^{\star \star \star}$ & $0.0018^{\star \star *}$ & $0.0036^{* * *}$ \\
\hline & $(0.0001)$ & $(0.0001)$ & $(0.0001)$ & $(0.0001)$ & $(0.0001)$ & $(0.0001)$ \\
\hline \multirow[t]{2}{*}{ Trabaja madre } & $-0.0049^{\star \star \star *}$ & 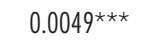 & $0.0083^{\star \star \star}$ & $0.0006^{\star \star \star}$ & $-0.0029 * \star \star$ & $0.0186^{\star \star \star}$ \\
\hline & $(0.0001)$ & $(0.0001)$ & $(0.0001)$ & $(0.0001)$ & $(0.0001)$ & $(0.0001)$ \\
\hline \multirow{2}{*}{$\begin{array}{l}\text { Número } \\
\text { de hiijos }\end{array}$} & $-0.0779^{\star \star \star \star}$ & $0.0243^{\star \star \star}$ & $0.0844^{\star \star \star}$ & $-0.1011^{\star \star \star}$ & $0.0626^{\star * \star}$ & $0.0194^{\star \star \star}$ \\
\hline & $(0.0007)$ & $(0.0009)$ & $(0.0001)$ & $(0.0008)$ & $(0.0011)$ & $(0.0011)$ \\
\hline \multirow{2}{*}{$\begin{array}{l}\text { Diferencia } \\
\text { de edad }\end{array}$} & $-0.0130^{\star \star \star}$ & $-0.0240^{\star \star \star}$ & $0.0078^{\star \star \star}$ & $0.0217^{\star \star \star}$ & $0.0399 * \star \star *$ & $0.0211^{\text {*** }}$ \\
\hline & $(0.0006)$ & $(0.0007)$ & $(0.0006)$ & $(0.0007)$ & $(0.0008)$ & $(0.0009)$ \\
\hline \multirow[t]{2}{*}{ Rural } & 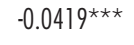 & $-0.0368^{\star \star \star}$ & $0.6268^{\star \star \star}$ & $-0.1147^{\star \star \star}$ & $-0.1635^{\star \star \star}$ & $0.2044^{\star \star \star}$ \\
\hline & $(0.0021)$ & $(0.0023)$ & $(0.0021)$ & $(0.0023)$ & $(0.0031)$ & $(0.0028)$ \\
\hline \multirow[t]{2}{*}{ Constante } & $30.933^{\star \star \star}$ & $-3.111^{\star \star \star}$ & $-4.567^{\star \star \star}$ & $4.511^{\star \star \star}$ & $-3.858^{\star \star \star}$ & $-3.919^{\star \star \star}$ \\
\hline & $(0.0114)$ & $(0.0187)$ & $(0.0132)$ & $(0.0134)$ & $(0.0160)$ & $(0.0171)$ \\
\hline Observaciones & & 23765 & & & 22848 & \\
\hline
\end{tabular}

Nota: ${ }^{\star} p<.10,{ }^{\star \star} p<.05,{ }^{\star \star \star} p<.01$. Errores estándar en paréntesis.

Fuente: Elaboración propia con base en datos del MTI-ENOE, 2011. 


\section{Pedro Orraca}

restricciones económicas. Por otra parte, la diferencia de edad entre hermanos tiene un efecto positivo sobre la asistencia escolar y la participación en el mercado laboral en el caso de las mujeres, mientras que para los hombres el efecto sólo es positivo en el caso del trabajo no retribuido. Finalmente, vivir en una localidad rural reduce la probabilidad de asistir a la escuela e incurrir en actividades renumeradas, e incrementa la probabilidad de tener un trabajo no pagado. Esto refleja la menor asistencia escolar en dichas comunidades, lo cual se debe, en parte, a la menor calidad de las escuelas en las zonas rurales y en casos extremos al acceso más difícil a las instalaciones educativas. Sin embargo, estos resultados deben de interpretarse con cautela debido a los diversos problemas de endogeneidad presentes en el modelo.

\section{Medidas para minimizar los problemas de endogeneidad}

En la estimación del modelo, la variable de fertilidad (i.e. número de hijos), presenta un serio problema de endogeneidad que surge a partir de un error de medición de la variable y la posibilidad de causalidad inversa, lo que genera estimadores sesgados e ineficientes que invalidan el análisis empírico.

El problema de error de medición de la variable de fertilidad se origina porque el regresor incluye, tanto a familias que ya dejaron de crecer y que, por lo tanto, no tendrán hijos adicionales, como aquellas que todavía se encuentran en proceso de formación (Emerson y Souza, 2008: 1651). Como no es posible observar para estas últimas el número final de hijos, se incurre en un problema de error de medición. De igual manera, dado que la variable contabiliza dos fenómenos distintos es altamente probable que presente un elevado grado de correlación con el término de error. Por otro lado, el problema de causalidad inversa se produce debido a que las decisiones de cuánto invertir en los hijos y cuántos hijos tener, son en general, determinadas de manera simultánea (Dammert, 2010: 200).

Comúnmente, estos problemas suelen solucionarse mediante el uso de variables instrumentales. Sin embargo, la falta de herramientas relevantes nos lleva a elegir un par de métodos alternativos. Con base en Emerson y Souza (2008), la primera estrategia consiste en limitar el análisis a los hogares donde las decisiones de fertilidad ya fueron tomadas y las familias están fijas en un cierto número de hijos. Para ello, se restringe la muestra a hogares donde la madre tiene más de 40 años y, por la tanto, hay bajas probabilidades de que en el futuro se tengan hijos adicionales. Para controlar el problema de causalidad inversa, se restringe la muestra a familias que tienen el mismo número de hijos, al enfocarse en las que tienen tres. Esto garantiza que todos los hogares incluidos en la muestra son insesgados, dado que no hay diferencias estructu- 
rales entre ellos. En caso de que se presenten discrepancias sistemáticas entre las familias con tres hijos y el resto de los hogares, es posible que los resultados no se puedan generalizar al resto de la población.

Las estimaciones del modelo probit multivariado para esta submuestra se presentan en el cuadro 5; donde se observa un alto grado de consistencia con respecto a los resultados anteriores. Tanto en el caso de los hombres como en el de las mujeres, el hijo más joven goza de una probabilidad mayor de asistir a la escuela respecto al resto de sus hermanos, mientras que para los varones primogénitos se presenta la situación opuesta y en el caso de las niñas ser la primogénita no está relacionado con la asistencia escolar. Referente al trabajo remunerado y el no pagado, se aprecia entre los varones que el hijo más chico es menos propenso a incurrir en ellos comparado al resto de sus hermanos. Asimismo, el trabajo no pagado es más común entre los varones más grandes del hogar. Por otro lado, las mujeres primogénitas tienen una probabilidad mayor de obtener un trabajo remunerado y una probabilidad menor de trabajar y no recibir ingresos.

Para intentar solucionar el problema de endogeneidad se propone una segunda estrategia basada en el método propuesto por Rosenzweig y Schultz (1987). El procedimiento consiste en estimar una regresión de fertilidad de la cual se obtienen sus residuales, para posteriormente introducirlos en el modelo como una variable exógena en sustitución del regresor número de hijos. ${ }^{6}$ La lógica detrás de esto radica en que, los residuales pueden ser interpretados como una medida de fecundidad natural, al fungir como un estimador del componente de fertilidad no explicado. Lo anterior surge porque si bien se puede pensar que los residuales están correlacionados con la fertilidad verdadera, difícilmente tendrán una relación con los términos de error de las regresiones de trabajo o asistencia escolar (Emerson y Souza, 2008:1652).

El cuadro 6 muestra los resultados de este modelo, donde se observa que las estimaciones son muy similares a las presentadas en el cuadro 4. Entre los resultados destaca que los varones más pequeños tienen una probabilidad mayor de asistir a la escuela respecto a sus hermanos primogénitos, pero una probabilidad menor de asistir en comparación con el resto de sus hermanos. En el caso de las mujeres, las hijas más pequeñas exhiben una mayor probabilidad de asistir a la escuela, mientras que las mayores tienden a incurrir menos en actividades escolares. Referente a las actividades laborales, los varones más pequeños son menos propensos a trabajar que sus hermanos primogénitos, independientemente de que se trate de trabajo remunerado o no. En el caso de las mujeres, las hijas primogénitas tienden a incurrir más en actividades de trabajo no remunerado, mientras que las últimas en nacer son más propensas a obtener empleos remunerados. 


\section{Pedro Orraca}

Cuadro 5. Resultados. Modelo probit multivariado. Muestra restringida a los hogares donde se tienen exactamente tres hijos y la madre tiene más de 40 años

\begin{tabular}{|c|c|c|c|c|c|c|}
\hline \multirow{2}{*}{$\begin{array}{l}\text { Edad } \\
\text { del niño }\end{array}$} & \multicolumn{3}{|c|}{ Hombres } & \multicolumn{3}{|c|}{ Mujeres } \\
\hline & $\begin{array}{c}\text { Asiste a la } \\
\text { escuela }\end{array}$ & $\begin{array}{l}\text { Trabajo } \\
\text { remunerado }\end{array}$ & $\begin{array}{l}\text { Trabajo no } \\
\text { remunerado }\end{array}$ & $\begin{array}{c}\text { Asiste a la } \\
\text { escuela }\end{array}$ & $\begin{array}{c}\text { Trabajo } \\
\text { remunerado }\end{array}$ & $\begin{array}{l}\text { Trabajo no } \\
\text { remunerado }\end{array}$ \\
\hline \multirow[t]{2}{*}{ Edad del niño } & $-0.2947^{\star \star \star}$ & $0.2437^{\star \star \star}$ & $0.1139^{\star \star \star \star}$ & $-0.3212^{\star \star \star}$ & $0.1672^{\star \star \star}$ & $0.1017^{\star \star \star}$ \\
\hline & $(0.0016)$ & $(0.0015)$ & $(0.0012)$ & $(0.0021)$ & $(0.0020)$ & $(0.0017)$ \\
\hline \multirow{2}{*}{$\begin{array}{l}\text { Niño más } \\
\text { chico }\end{array}$} & $0.2004^{\star \star \star}$ & $-0.3653^{\star \star \star}$ & $-0.1144^{\star \star \star}$ & $0.2482^{\star \star \star}$ & $0.0757^{\star \star \star}$ & $-0.2956^{\star \star \star}$ \\
\hline & $(0.0065)$ & $(0.0064)$ & $(0.0069)$ & $(0.0080)$ & $(0.0100)$ & $(0.0095)$ \\
\hline \multirow{2}{*}{$\begin{array}{l}\text { Niño más } \\
\text { grande }\end{array}$} & $-0.1598^{\star \star \star}$ & $-0.2787^{\star \star \star}$ & $0.0832^{\star \star \star}$ & 0.0062 & $0.1317^{\star \star \star}$ & $-0.3038^{\star \star \star \star}$ \\
\hline & $(0.0076)$ & $(0.0077)$ & $(0.0084)$ & $(0.0089)$ & $(0.0122)$ & $(0.0133)$ \\
\hline \multirow{2}{*}{$\begin{array}{l}\text { Escolaridad } \\
\text { padre }\end{array}$} & $0.0804^{\star \star \star}$ & $-0.0294^{\star \star \star}$ & 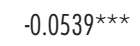 & $0.0691^{\star * \star}$ & $-0.0594^{\star \star \star}$ & $-0.0107^{\star \star \star}$ \\
\hline & $(0.0006)$ & $(0.0119)$ & $(0.0007)$ & $(0.0007)$ & $(0.0010)$ & $(0.0010)$ \\
\hline \multirow{2}{*}{$\begin{array}{l}\text { Escolaridad } \\
\text { madre }\end{array}$} & $0.0280^{\star * \star *}$ & $-0.0089^{\star \star \star \star}$ & $-0.0087^{\star \star \star}$ & $0.0210^{\star \star \star *}$ & $0.0170^{\star \star \star}$ & $0.0305^{\star \star \star}$ \\
\hline & $(0.0008)$ & $(0.0007)$ & $(0.0008)$ & $(0.0008)$ & $(0.0010)$ & $(0.0010)$ \\
\hline \multirow[t]{2}{*}{ Edad padre } & $0.0084^{\star \star \star}$ & 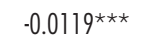 & 0.0006 & $0.0070^{\star \star \star}$ & $0.0380^{\star \star \star}$ & 0.0006 \\
\hline & $(0.0005)$ & $(0.0006)$ & $(0.0005)$ & $(0.0006)$ & $(0.0008)$ & $(0.0009)$ \\
\hline \multirow[t]{2}{*}{ Edad madre } & $-0.0080^{\star \star \star}$ & $-0.0184^{\star \star \star}$ & $0.0026^{\star \star \star}$ & $-0.0317^{\star \star \star}$ & $-0.0678^{\star \star \star}$ & 0.0003 \\
\hline & $(0.0007)$ & $(0.0009)$ & $(0.0007)$ & $(0.0010)$ & $(0.0014)$ & $(0.0014)$ \\
\hline \multirow[t]{2}{*}{ Trabaja padre } & $-0.0064^{\star \star \star}$ & $0.0013^{\star \star \star}$ & $0.0106^{\star \star \star}$ & $0.0088^{\star * \star}$ & $0.0070^{\star \star \star}$ & $0.0046^{\star \star *}$ \\
\hline & $(0.0001)$ & $(0.0001)$ & $(0.0001)$ & $(0.0001)$ & $(0.0002)$ & $(0.0002)$ \\
\hline \multirow[t]{2}{*}{ Trabaja madre } & $-0.0018^{\star \star \star}$ & $0.0044^{\star \star \star}$ & $0.0121^{\star \star \star}$ & $0.0013^{\star \star \star}$ & $-0.0013^{\star \star \star}$ & $0.0186^{\text {*** }}$ \\
\hline & $(0.0001)$ & $(0.0001)$ & $(0.0001)$ & $(0.0002)$ & $(0.0002)$ & $(0.0002)$ \\
\hline \multirow{2}{*}{$\begin{array}{l}\text { Diferencia } \\
\text { de edad }\end{array}$} & $-0.0515^{\star \star \star}$ & $-0.0720^{\star \star \star}$ & $0.0547^{\star \star \star}$ & $0.0634^{\star \star \star}$ & $-0.0593^{\star \star \star}$ & $0.0231^{\star \star \star}$ \\
\hline & $(0.0020)$ & $(0.0022)$ & $(0.0020)$ & $(0.0022)$ & $(0.0032)$ & $(0.0031)$ \\
\hline \multirow[t]{2}{*}{ Rural } & $-0.2505^{\star \star \star}$ & $0.1296^{\star \star \star}$ & $0.6106^{\star \star \star *}$ & $0.0772^{\star \star \star}$ & $-0.3690^{\star \star \star}$ & $0.6323^{\star \star \star}$ \\
\hline & $(0.0073)$ & $(0.0073)$ & $(0.0070)$ & $(0.0081)$ & $(0.0108)$ & $(0.0100)$ \\
\hline \multirow[t]{2}{*}{ Constante } & $4.868^{\star \star \star}$ & $-3.403^{\star \star \star}$ & $-4.144^{\star \star \star}$ & $6.113^{\star \star \star *}$ & $-3.171^{\star \star \star}$ & $-4.763^{\text {*** }}$ \\
\hline & $(0.0402)$ & $(0.0520)$ & $(0.0450)$ & $(0.0507)$ & $(0.0762)$ & $(0.0778)$ \\
\hline Observaciones & & 2528 & & & 2048 & \\
\hline
\end{tabular}

Nota: ${ }^{\star} p<.10,{ }^{\star \star} p<.05,{ }^{\star \star \star} p<.01$. Errores estándar en paréntesis.

Fuente: Elaboración propia con base en datos del MTI-ENOE, 2011. 
Cuadro 6. Resultados. Modelo probit multivariado. Muestra completa estimada con los residuales de una regresión de fertilidad

\begin{tabular}{|c|c|c|c|c|c|c|}
\hline \multirow{2}{*}{$\begin{array}{l}\text { Edad } \\
\text { del niño }\end{array}$} & \multicolumn{3}{|c|}{ Hombres } & \multicolumn{3}{|c|}{ Mujeres } \\
\hline & $\begin{array}{c}\text { Asiste a la } \\
\text { escuela }\end{array}$ & $\begin{array}{c}\text { Trabajo } \\
\text { remunerado }\end{array}$ & $\begin{array}{l}\text { Trabajo no } \\
\text { remunerado }\end{array}$ & $\begin{array}{c}\text { Asiste a la } \\
\text { escuela }\end{array}$ & $\begin{array}{l}\text { Trabajo } \\
\text { remunerado }\end{array}$ & $\begin{array}{l}\text { Trabajo no } \\
\text { remunerado }\end{array}$ \\
\hline \multirow[t]{2}{*}{ Edad del niño } & $-0.2183^{\star \star \star}$ & $0.1781^{\star \star \star}$ & $0.1156^{\star \star \star}$ & $-0.2326^{\star \star \star \star}$ & $0.1533^{\star \star \star}$ & 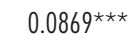 \\
\hline & $(0.0004)$ & $(0.0003)$ & $(0.0003)$ & $(0.0004)$ & $(0.0005)$ & $(0.0004)$ \\
\hline \multirow{2}{*}{$\begin{array}{l}\text { Niño más } \\
\text { chico }\end{array}$} & $-0.0585^{\star \star \star}$ & $-0.0700^{\star \star \star}$ & $0.0159^{\star \star \star}$ & $0.0081^{\star \star \star}$ & $0.0756^{\star \star \star}$ & $-0.0754^{\star \star \star}$ \\
\hline & $(0.0026)$ & $(0.0027)$ & $(0.0026)$ & $(0.0030)$ & $(0.0036)$ & $(0.0035)$ \\
\hline \multirow{2}{*}{$\begin{array}{l}\text { Niño más } \\
\text { grande }\end{array}$} & $-0.0592^{\star \star \star}$ & $0.0305^{\star \star \star}$ & $0.1547^{\star \star \star}$ & $-0.1646^{\star \star \star}$ & $0.0719^{\star \star \star}$ & $0.1291^{\star \star \star}$ \\
\hline & $(0.0022)$ & $(0.0023)$ & $(0.0023)$ & $(0.0024)$ & $(0.0030)$ & $(0.0031)$ \\
\hline \multirow{2}{*}{$\begin{array}{l}\text { Escolaridad } \\
\text { padre }\end{array}$} & $0.0876^{\star \star \star \star}$ & 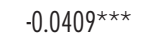 & $-0.0463^{\star \star \star}$ & $0.0929^{\star \star \star *}$ & $-0.0435^{\star \star \star}$ & $-0.0396^{\text {*** }}$ \\
\hline & $(0.0002)$ & $(0.0002)$ & $(0.0002)$ & $(0.0002)$ & $(0.0003)$ & $(0.0003)$ \\
\hline \multirow{2}{*}{$\begin{array}{l}\text { Escolaridad } \\
\text { madre }\end{array}$} & $0.0322^{\star \star \star}$ & $-0.0017^{\star \star \star}$ & $0.0149^{\star \star \star *}$ & $0.0244^{\star \star \star}$ & $0.0135^{\star \star \star}$ & $0.0243^{\star \star \star}$ \\
\hline & $(0.0003)$ & $(0.0002)$ & $(0.0002)$ & $(0.0003)$ & $(0.0003)$ & $(0.0003)$ \\
\hline \multirow[t]{2}{*}{ Edad padre } & $0.0034^{\star \star \star}$ & $-0.0099 * \star \star *$ & $0.0068^{\star \star \star}$ & $0.0021^{\star \star \star}$ & $-0.0016^{\star \star \star}$ & $0.0122^{\star \star *}$ \\
\hline & $(0.0001)$ & $(0.0001)$ & $(0.0001)$ & $(0.0001)$ & $(0.0002)$ & $(0.0002)$ \\
\hline \multirow[t]{2}{*}{ Edad madre } & 0.0001 & $-0.0023^{\star \star \star}$ & $0.0010^{* \star *}$ & $-0.0036^{\star \star \star \star}$ & $-0.0078^{\star \star \star}$ & $-0.0140^{\star \star \star *}$ \\
\hline & $(0.0002)$ & $(0.0002)$ & $(0.0002)$ & $(0.0002)$ & $(0.0002)$ & $(0.0002)$ \\
\hline \multirow[t]{2}{*}{ Trabaja padre } & $-0.0022^{\star \star \star}$ & $0.0008^{\star \star \star}$ & $0.0075^{\star \star \star}$ & $-0.0014^{\star \star \star}$ & $0.0018^{\star \star \star}$ & $0.0036^{\star \star \star *}$ \\
\hline & $(0.0001)$ & $(0.0001)$ & $(0.0001)$ & $(0.0001)$ & $(0.0001)$ & $(0.0001)$ \\
\hline \multirow[t]{2}{*}{ Trabaja madre } & 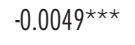 & $0.0049^{\star * \star}$ & $0.0083^{\star \star \star}$ & $-0.0006^{* \star \star}$ & $0.0029 * \star \star$ & $0.0186^{\star \star \star *}$ \\
\hline & $(0.0001)$ & $(0.0001)$ & $(0.0001)$ & $(0.0001)$ & $(0.0001)$ & $(0.0001)$ \\
\hline \multirow[t]{2}{*}{ Residuales } & 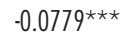 & $0.0243^{\star \star \star}$ & $0.0844^{\star \star \star}$ & $-0.1011^{\star \star \star}$ & $0.0626^{\star \star *}$ & $0.0194^{\star \star \star}$ \\
\hline & $(0.0007)$ & $(0.0009)$ & $(0.0008)$ & $(0.0008)$ & $(0.0011)$ & $(0.0011)$ \\
\hline \multirow{2}{*}{$\begin{array}{l}\text { Diferencia } \\
\text { de edad }\end{array}$} & $-0.0130^{\star \star \star}$ & $-0.0240^{\star \star \star}$ & $0.0078^{\star \star \star}$ & $0.0217^{\star \star \star}$ & $0.0339 * \star \star$ & $0.0211^{\star * *}$ \\
\hline & $(0.0006)$ & $(0.0007)$ & $(0.0006)$ & $(0.0007)$ & $(0.0008)$ & $(0.0009)$ \\
\hline \multirow[t]{2}{*}{ Rural } & $-0.0771^{\star \star \star}$ & $-0.0258^{\star * *}$ & 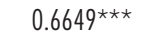 & $-0.1604^{\star \star \star}$ & $-0.1353^{\star \star \star}$ & $0.2132^{\star \star \star}$ \\
\hline & $(0.0021)$ & $(0.0023)$ & $(0.0021)$ & $(0.0023)$ & $(0.0031)$ & $(0.0028)$ \\
\hline \multirow[t]{2}{*}{ Constante } & $3.671^{\star \star \star}$ & $-3.169 * \star \star$ & $-4.283^{\star \star \star}$ & $4.170^{\star \star \star}$ & $-0.3648 * \star \star *$ & $-3.854^{\star \star \star}$ \\
\hline & $(0.0109)$ & $(0.0115)$ & $(0.0128)$ & $(0.0128)$ & $(0.0153)$ & $(0.0165)$ \\
\hline Observaciones & & 23765 & & & 22848 & \\
\hline
\end{tabular}

Nota: ${ }^{\star} p<.10,{ }^{\star \star} p<.05,{ }^{\star \star \star} p<.01$. Errores estándar en paréntesis.

Fuente: Elaboración propia con base en datos del MTI-ENOE, 2011. 
La última estrategia empírica consiste en reclasificar la muestra, donde sólo aquellos niños o adolescentes que laboran, porque el hogar afirma que requiere de su trabajo o aportación económica, son considerados trabajadores infantiles. Lo anterior sugiere que estas familias introducen a sus hijos en el mercado laboral ya que enfrentan fuertes restricciones presupuestarias. Debido a su carencia de recursos para enviar al primogénito a la escuela, y dada su mayor capacidad de ingresos y facilidad en encontrar una ocupación, se espera que en estos hogares que se encuentran ante una situación de pobreza los hijos de mayor edad tengan más propensión hacia el trabajo.

En las estimaciones que se presentan en el cuadro 7 se puede observar la misma dirección en los resultados a los que se encontraron previamente. Sin embargo, las magnitudes entre los coeficientes se han acentuado de tal manera que hay una mayor diferencia entre las actividades de los hijos de acuerdo a su orden de nacimiento. Esto sugiere que la pobreza y las restricciones presupuestarias desempeñan un papel significativo en las decisiones de asignación de tiempo de los hogares.

Cuadro 7. Resultados. Modelo probit multivariado. Muestra reclasificada donde sólo aquellos menores que laboran porque el hogar requiere de su aportación son considerados trabajadores

\begin{tabular}{|c|c|c|c|c|c|c|}
\hline \multirow{2}{*}{$\begin{array}{l}\text { Edad } \\
\text { del niño }\end{array}$} & \multicolumn{3}{|c|}{ Hombres } & \multicolumn{3}{|c|}{ Mujeres } \\
\hline & $\begin{array}{c}\text { Asiste a la } \\
\text { escuela }\end{array}$ & $\begin{array}{l}\text { Trabajo } \\
\text { remunerado }\end{array}$ & $\begin{array}{l}\text { Trabajo no } \\
\text { remunerado }\end{array}$ & $\begin{array}{c}\text { Asiste a la } \\
\text { escuela }\end{array}$ & $\begin{array}{c}\text { Trabajo } \\
\text { remunerado }\end{array}$ & $\begin{array}{l}\text { Trabajo no } \\
\text { remunerado }\end{array}$ \\
\hline \multirow[t]{2}{*}{ Edad del niño } & $-0.2340^{\star \star \star \star}$ & $0.2064^{\star \star \star}$ & $0.1236^{\star \star \star}$ & $-0.2354^{\star \star \star}$ & $0.1861^{\star \star \star}$ & 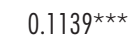 \\
\hline & $(0.0004)$ & $(0.0010)$ & $(0.0004)$ & $(0.0004)$ & $(0.0015)$ & $(0.0006)$ \\
\hline \multirow{2}{*}{$\begin{array}{l}\text { Niño más } \\
\text { chico }\end{array}$} & $-0.0549^{* \star \star}$ & $-0.1505^{\star \star \star}$ & $0.0759^{\star \star *}$ & $0.1222^{\star \star \star *}$ & $0.0703^{\star \star \star}$ & $-0.1671^{\star \star \star}$ \\
\hline & $(0.0023)$ & $(0.0060)$ & $(0.0033)$ & $(0.0030)$ & $(0.0082)$ & $(0.0045)$ \\
\hline \multirow{2}{*}{$\begin{array}{l}\text { Niño más } \\
\text { grande }\end{array}$} & $-0.0760^{\star \star \star \star}$ & $0.0918^{\star \star \star}$ & $0.1851^{\star \star \star}$ & $-0.1613^{\star \star \star}$ & $0.0541^{\star \star \star}$ & $0.1665^{\star \star \star}$ \\
\hline & $(0.0026)$ & $(0.0046)$ & $(0.0029)$ & $(0.0122)$ & $(0.0068)$ & $(0.0038)$ \\
\hline \multirow{2}{*}{$\begin{array}{l}\text { Escolaridad } \\
\text { padre }\end{array}$} & $0.0840^{\star \star \star}$ & $-0.0506^{\star \star \star}$ & $-0.0513^{\star \star \star}$ & $0.0879^{\star \star \star *}$ & $-0.0397^{\star \star \star}$ & $-0.0468^{\star \star \star *}$ \\
\hline & $(0.0002)$ & $(0.0005)$ & $(0.0003)$ & $(0.0002)$ & $(0.0007)$ & $(0.0004)$ \\
\hline \multirow{2}{*}{$\begin{array}{l}\text { Escolaridad } \\
\text { madre }\end{array}$} & $0.0326^{\star \star \star}$ & $-0.0166^{\star \star \star}$ & $0.0205^{\star \star \star}$ & $0.0227^{\star \star \star}$ & $-0.0033^{\star \star \star}$ & $0.0299 * * \star$ \\
\hline & $(0.0003)$ & $(0.0006)$ & $(0.0002)$ & $(0.0003)$ & $(0.0008)$ & $(0.0004)$ \\
\hline \multirow[t]{2}{*}{ Edad padre } & $0.0043^{\star \star \star *}$ & 0.0004 & $0.0026^{\star \star * \star}$ & 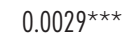 & $-0.0067^{\star \star \star}$ & $0.0120^{* \star *}$ \\
\hline & $(0.0001)$ & $(0.0003)$ & $(0.0002)$ & $(0.0001)$ & $(0.0004)$ & $(0.0002)$ \\
\hline
\end{tabular}




\begin{tabular}{|c|c|c|c|c|c|c|}
\hline \multirow{2}{*}{$\begin{array}{l}\text { Edad } \\
\text { del niño }\end{array}$} & \multicolumn{3}{|c|}{ Hombres } & \multicolumn{3}{|c|}{ Mujeres } \\
\hline & $\begin{array}{c}\text { Asiste a la } \\
\text { escuela }\end{array}$ & $\begin{array}{l}\text { Trabajo } \\
\text { remunerado }\end{array}$ & $\begin{array}{l}\text { Trabajo no } \\
\text { remunerado }\end{array}$ & $\begin{array}{c}\text { Asiste a la } \\
\text { escuela }\end{array}$ & $\begin{array}{l}\text { Trabajo } \\
\text { remunerado }\end{array}$ & $\begin{array}{l}\text { Trabajo no } \\
\text { remunerado }\end{array}$ \\
\hline \multirow[t]{2}{*}{ Edad madre } & 0.0001 & $-0.0193^{\star \star \star}$ & $0.0017^{\star \star \star *}$ & $-0.0042^{\star \star \star}$ & $0.0102^{\star \star \star}$ & $-0.0116^{\star \star \star *}$ \\
\hline & $(0.0002)$ & $(0.0004)$ & $(0.0002)$ & $(0.0002)$ & $(0.0006)$ & $(0.0003)$ \\
\hline \multirow[t]{2}{*}{ Trabaja padre } & $-0.0023^{\star \star \star}$ & $-0.0004^{\star \star \star}$ & $0.0082^{\star \star \star}$ & $-0.0014^{\star \star \star}$ & $-0.0026^{\star \star \star}$ & $0.0018^{\star \star \star *}$ \\
\hline & $(0.0001)$ & $(0.0001)$ & $(0.0001)$ & $(0.0001)$ & $(0.0001)$ & $(0.0001)$ \\
\hline \multirow[t]{2}{*}{ Trabaja madre } & $-0.0048^{\star \star \star}$ & $0.0074^{\star * \star}$ & $0.0102^{\star \star \star *}$ & $-0.0007^{\star \star \star}$ & $0.0085^{\star \star \star}$ & $0.0189 \star \star \star *$ \\
\hline & $(0.0001)$ & $(0.0001)$ & $(0.0001)$ & $(0.0001)$ & $(0.0001)$ & $(0.0001)$ \\
\hline \multirow{2}{*}{$\begin{array}{l}\text { Número de } \\
\text { hiijos }\end{array}$} & $-0.0833^{\star \star \star *}$ & $0.0692^{\star \star \star}$ & $0.1054^{\star \star \star}$ & $-0.1007^{\star \star \star \star}$ & $0.1060^{\star \star \star}$ & $0.0292^{\star \star \star *}$ \\
\hline & $(0.0008)$ & $(0.0016)$ & $(0.0009)$ & $(0.0008)$ & $(0.0022)$ & $(0.0014)$ \\
\hline \multirow{2}{*}{$\begin{array}{l}\text { Diferencia } \\
\text { de edad }\end{array}$} & $-0.0125^{\star \star \star}$ & $-0.0516^{\star \star \star}$ & $0.0243^{\star \star \star}$ & $0.0203^{\star \star \star}$ & $-0.0066^{\star \star \star}$ & $0.0426^{\star \star \star}$ \\
\hline & $(0.0006)$ & $(0.0015)$ & (0.0008) & $(0.0007)$ & $(0.0020)$ & $(0.0011)$ \\
\hline \multirow[t]{2}{*}{ Rural } & $-0.0534^{\star \star \star}$ & $0.1596^{\star \star \star}$ & $0.6253^{\star \star \star}$ & 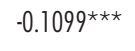 & $0.2816^{\star \star \star}$ & $0.1986^{\star \star \star}$ \\
\hline & $(0.0021)$ & $(0.0043)$ & $(0.0027)$ & $(0.0023)$ & $(0.0063)$ & $(0.0035)$ \\
\hline \multirow[t]{2}{*}{ Constante } & $4.130^{\star \star \star}$ & $-4.174^{\star \star \star}$ & $-4.970^{\star \star \star \star}$ & $4.537^{\star \star \star}$ & $-5.827^{\star \star \star}$ & $-4.701^{\star \star \star}$ \\
\hline & $(0.0117)$ & $(0.0246)$ & $(0.0168)$ & $(0.0134)$ & $(0.0449)$ & $(0.0233)$ \\
\hline Observaciones & & 23765 & & & 22848 & \\
\hline
\end{tabular}

Nota: ${ }^{*} p<.10,{ }^{\star \star} p<.05,{ }^{* \star \star} p<.01$. Errores estándar en paréntesis.

Fuente: Elaboración propia con base en datos del MTI-ENOE, 2011.

\section{CONCLUSIONES}

El presente artículo estudió el efecto del orden de nacimiento de los hijos sobre la asignación del tiempo destinado a la asistencia escolar y la participación en actividades laborales remuneradas y las no pagadas en México. Para ello se emplearon datos del MTI y se estimó un modelo probit multivariado que toma en cuenta la interrelación entre estas tres tareas. Los resultados muestran que los hijos primogénitos tienen una probabilidad menor de asistir a la escuela y son más proclives a trabajar, comparado con el resto de sus hermanos. Por otro lado, los hijos más jóvenes gozan de una inclinación mayor a asistir a la escuela, y en el caso de las mujeres, una propensión menor a incurrir en actividades de trabajo remunerado respecto a sus hermanos más grandes. Las 


\section{Pedro Orraca}

relaciones encontradas se acentúan al enfocarnos en los hogares donde los hijos laboran debido a la falta de recursos. El estudio proporciona evidencia de que hay diferencias importantes en la asignación de tiempo de las actividades de los hijos de acuerdo a su orden de nacimiento.

Por otra parte, para minimizar el problema del trabajo infantil en México, se requiere de una aplicación más estricta de las leyes que impiden la contratación de trabajadores infantiles, y una mayor regulación que garantice condiciones laborales favorables y flexibles para aquellos menores que se encuentran dentro de la edad legal para trabajar. Se deben de instrumentar programas que desincentiven el uso del trabajo infantil y promuevan la asistencia escolar, tomando en cuenta no solamente el estatus económico del hogar sino también su composición. Sin embargo, ante todo, se requiere de un desarrollo sostenido de la economía que eleve el ingreso real de los hogares, para que éstos no se vean ante la necesidad de insertar a sus hijos menores de edad en el mercado laboral y el trabajo infantil sólo surja como una elección y no como producto de la falta de recursos.

Finalmente, es importante recordar que si bien el trabajo infantil tiende a ser nocivo, hay casos excepcionales donde puede ser benéfico, siempre y cuando no interfiera con la asistencia y las tareas escolares, ya que tiene el potencial de promover el desarrollo físico y mental de los menores. Por ello, una política mal diseñada destinada a eliminar el trabajo infantil que no tome en cuenta la generación de posibles externalidades que esto conllevaría, puede llegar a incrementar los niveles de pobreza en los que viven estos hogares, agravando todavía más la situación inicial.

\section{NOTAS}

1 La literatura que explica las razones por las cuales surge el trabajo infantil es extensa, de ésta destaca Ranjan (2001) el cual expone que el trabajo infantil es producto de restricciones crediticias e inequidades en la distribución de ingresos. Por otro lado, Hazan y Berdugo (2002) aseveran que el trabajo infantil es copioso cuando la economía se encuentra en un nivel de desarrollo bajo. Sin embargo, el progreso tecnológico aumenta la brecha salarial entre trabajadores adultos e infantiles, llevando a que los padres sustituyan el trabajo infantil por educación. Para una revisión de la literatura véase Basu (1999).

2 Esta definición potencialmente excluye a las personas más vulnerables de la sociedad, como son los menores de edad que ofrecen sus productos y servicios en la vía pública o en los transportes públicos (INEGI, 2012). También descarta a los niños y adolescentes que habitan en hogares sin padres. 
3 De manera específica, en este estudio $y_{i, 1}=1$ si el menor asiste a la escuela, 0 en otro caso; $y_{i, 2}$ $=1$ si el menor incurre en trabajo remunerado, 0 en otro caso y, por último, $y_{i, 3}=1$ si el menor incurre en trabajo no remunerado, 0 en otro caso.

4 Para una discusión más a detalle sobre el método de estimación y el programa estadístico utilizado véanse Cappellari y Jenkins (2003).

5 Todas las regresiones presentadas en este estudio incluyen variables dicotómicas que indican la entidad federativa de residencia y fueron estimadas utilizando errores estándar robustos basados en White (1980).

6 Concretamente, se estima una regresión de fertilidad por el método de MCO con errores estándar robustos basados en White (1980), donde la variable dependiente está dada por el número de hijos. El vector de variables exógenas está compuesto por los regresores edad del padre, de la madre, nivel educativo del padre, de la madre y una variable dicotómica que indica si el hogar pertenece a una localidad rural.

\section{BIBLIOGRAFÍA}

Baland, Jean, y James Robinson (2000), "Is child labor inefficient?", Journal of Political Economy, vol. 108, núm. 4, Chicago, University of Chicago Press, julio, pp. 663-679.

Bando, Rosángela; Luis López-Calva et al. (2005), "Child labor, school attendance, and indigenous households: Evidence from Mexico", Banco Mundial, working paper, núm. 3487, Washington.

Basu, Kaushik, y Pham Van (1998), "The economics of child labor", American Economic Review, vol. 88, núm. 3, Nashville, American Economic Association, junio, pp. 412-427.

Basu, Kaushik (1999), "Child labor: Cause, consequence, and cure, with remarks on international labor standards", Journal of Economic Literature, Nashville, American Economic Association, septiembre, vol. 37, núm. 3, pp. 1083-1119.

Becker, Gary (1991), A treatise on the family, Boston, Harvard University Press, pp. 304.

Börsch-Supan, Axel, y Vassilis Hajivassiliou (1993), "Unbiased multivariate probability simulators for maximum likelihood estimation of limited dependent variable models", Journal of Econometrics, vol. 58 núm. 3, Amsterdam, Elsevier, agosto, pp. 347-368.

Cappellari, Lorenzo, y Stephen Jenkins (2003), "Multivariate probit regression using simulated maximum likelihood”, Stata Journal, vol. 3, núm. 3, College Station, Stata Press, primer trimestre, pp. 278-294. 
Cigno, Alessandro, y Furio Rosati (2005), The economics of child labour, Oxford, Oxford University Press, pp. 264.

Córdova, Karina (2009), "The effect of birth order on education levels and time allocation decisions: Evidence from Mexican households", mimeo. Tucson, University of Arizona.

Constitución Política de los Estados Unidos Mexicanos (2008), México, Tribunal Electoral del Poder Judicial de la Federación.

Dammert, Ana (2010), "Siblings, child labor, and schooling in Nicaragua and Guatemala", Journal of Population Economics, vol. 23, núm. 1, Berlín, enero, pp. 199-224.

De Haan, Monique; Erik Plug et al. (2012), "Birth order and human capital development: Evidence from Ecuador”, IZA, discussion paper, núm. 6706, Bonn, IZA.

Emerson, Patrick, y André Souza (2008), "Birth order, child labor, and school attendance in Brazil", World Development, vol. 36, núm. 9, Amsterdam, Elsevier, agosto, pp. 1647-1664.

Hazan, Moshe, y Binyamin Berdugo (2002), "Child labour, fertility and economic growth", Economic Journal, vol, 112, núm. 482, Londres, RES, octubre, pp. 810-828.

Heckman, James (1979), "Sample selection bias as a specification error", Econometrica, vol. 47, núm. 1, Nueva Jersey, Wiley, enero, pp. 153-161.

INEGi (Instituto Nacional de Geografía y Estadística) (2012), "Módulo de Trabajo Infantil 2011, Encuesta Nacional de Ocupación y Empleo", Aguascalientes, disponible en: <http://www.inegi.org.mx/est/contenidos/ proyectos/encuestas/hogares/modulos/mti/mti2011/default.aspx $>$.

Keane, Michael (1994), "A computationally practical simulation estimator for panel data", Econometrica, vol. 62, núm. 1, Nueva Jersey, Wiley, enero, pp. 95-116.

Levison, Deborah; Karine Moe et al. (2001), "Youth education and work in Mexico”, World Development, vol. 29, núm. 1, Amsterdam, Elsevier, enero, pp. 167-188.

López-Calva, Luis, y Samuel Freije (2001), "Child labor, school attendance, and poverty in Mexico and Venezuela", Centro de Estudios Económicos, documento de trabajo, núm. 1, México, Colegio de México.

López, Antonia (2005), "Schooling and child labour in Mexico: An empirical analysis", Análisis Económico, vol. 20, núm. 45, México, UAM, tercer cuatrimestre, pp. 89-117.

OIT (Organización Internacional del Trabajo) (2010), Accelerating action against child labour, Ginebra, oIT, pp. 98. 
Ranjan, Priya (2001), "Credit constraints and the phenomenon of child labor", Journal of Development Economics, vol. 64, núm. 1, Amsterdam, Elsevier, febrero, pp. 81-102.

Rosenzweig, Mark, y Paul Schultz (1987), "Fertility and investments in human capital: Estimates of the consequence of imperfect fertility control in Malaysia”, Journal of Econometrics, vol. 36, núms. 1-2, Amsterdam, Elsevier, septiembre-octubre, pp. 163-184.

Skoufias, Emmanuel, y Susan Parker (2001), "Conditional cash transfers and their impact on child work and schooling: Evidence from the Progresa program in Mexico", Economia, vol. 2, núm. 1, Washington, Brookings Institution Press, otońo, pp. 45-86.

White, Halbert (1980), "A heteroskedasticity-consistent covariance matrix estimator and a direct test for heteroskedasticity", Econometrica, vol. 48, núm. 4, Nueva Jersey, Wiley, mayo, pp. 817-838. 
